\title{
Imaging diagnosis for chronic lateral ankle ligament injury: a systemic review with meta-analysis
}

\author{
Shengxuan $\mathrm{CaO}^{\dagger}$, Chen Wang ${ }^{\dagger}$, Xin Ma ${ }^{*}$, Xu Wang, Jiazhang Huang and Chao Zhang
}

\begin{abstract}
Background: Various imaging techniques have been utilized for the diagnosis of chronic lateral ankle ligament injury. This systemic review will explore the effectiveness of different imaging techniques in diagnosing chronic lateral ankle ligament injury.

Methods: Relative studies were retrieved after searching 3 databases (MEDLINE, EMBASE, and Cochrane Central Register of Controlled Trails). Eligible studies were summarized. Data were extracted to calculate pooled sensitivity and specificity of magnetic resonance imaging (MRI), ultrasonography (US), stress radiography, and arthrography.

Results: Fifteen studies met our inclusion and exclusion criteria. A total of 695 participants were included. The pooled sensitivities in diagnosing chronic ATFL injury were $0.83[0.78,0.87]$ for MRI, $0.99[0.96,1.00]$ for US, and 0.81 $[0.68,0.90]$ for stress radiography. The pooled specificities in diagnosing chronic ATFL injury were $0.79[0.69,0.87]$ for MRI, $0.91[0.82,0.97]$ for US, and $0.92[0.79,0.98]$ for stress radiography. The pooled sensitivities in diagnosing chronic CFL injury were $0.56[0.46,0.66]$ for MRI, $0.94[0.85,0.98]$ for US, and $0.90[0.73,0.98]$ for arthrography. The pooled specificities in diagnosing chronic CFL injury were 0.88 [0.82, 0.93] for MRI, $0.91[0.80,0.97]$ for US, and 0.90 $[0.77,0.97]$ for arthrography.

Conclusion: This systematic review with meta-analysis investigated the accuracy of imaging for the diagnosis of chronic lateral ankle ligament injury. Ultrasound manifested high diagnostic accuracy in diagnosing chronic lateral ankle ligament injury. Clinicians should be aware of the limitations of MRI in detecting chronic CFL injuries.
\end{abstract}

Keywords: Ankle ligaments, Meta-analysis, Imaging, Diagnostic accuracy

\section{Background}

Ankle sprain is one of the most common sports injuries [1-3]. The injury mechanism, a combination of inversion and adduction in foot plantar flexion, can cause damage to the lateral ankle ligaments [4]. Lateral ankle ligaments comprise anterior talofibular ligament (ATFL), calcaneofibular ligament (CFL), and posterior talofibular ligament (PTFL) [5]. Chronic lateral ankle ligament injury is a long-term outcome in patients suffering acute ankle sprain. Some long-term cohort studies showed that 10 to $20 \%$ patients eventually developed persistent symptoms, including pain, swelling, perception of instability, and recurrent ankle

\footnotetext{
*Correspondence: prof.xin.ma@qq.com

†Shengxuan Cao and Chen Wang contributed equally to this work. Department of Orthopedics, Huashan Hospital, Fudan University, No.12, Middle Wulumuqi Road, Jingan District, Shanghai, China
}

sprain [6-9]. The precise cause of these symptoms is still in debate and probably multifactorial $[5,10-12]$. But identifying chronic lateral ankle ligament injury is critical for locating primal symptoms, and thus, the decision making of surgical intervention [13].

Compared to acute ligament injury, the proper diagnosis of chronic ligament injury is difficult because of the uncertain association between initial inversion trauma history and ligament injury and diversified manifestation of chronic injuries on images [14, 15]. The patients' history and clinical tests are important in diagnosis. A meta-analysis discussed the accuracy of clinical tests in diagnosing ankle ligament injury and claimed that clinicians cannot rule out ligamentous injury when physical tests are negative, and additional imaging is warranted [16]. Imaging is a helpful diagnostic method according to consensus of experts [17, 18].

(C) The Author(s). 2018 Open Access This article is distributed under the terms of the Creative Commons Attribution 4.0 International License (http://creativecommons.org/licenses/by/4.0/), which permits unrestricted use, distribution, and 
However, different from acute injury, chronic ligament injury could show as either stretching, rupture, scarring, or thickening of the ligament on images, which confuses the clinical practitioners [19]. Magnetic resonance imaging (MRI), ultrasonography (US), stress radiography, and arthrography are often utilized for diagnosing chronic lateral ankle ligament injury. Separate studies on various imaging diagnostic techniques have been reported; however, the diagnostic effectiveness of different imaging techniques was still controversial, partly due to different existing reference standards [20, 21].

A previous systematic review assessed US for diagnosis of chronic ankle instability, but utilized variable gold standards, and did not provide pooled data [20]. Studies of MRI, US, stress radiography, and arthrography for diagnosis of chronic lateral ankle ligament injury were reported, but no previous systemic review provided synthesized data. This systemic review with meta-analysis is trying to analyze studies on diagnostic accuracy of different imaging techniques on chronic lateral ligament injury. Arthroscopic or surgical findings are set as the gold standards of ligament injuries [12, 22].

\section{Methods}

\section{Inclusion and exclusion criteria}

The studies that met the following criteria were included: (1) cohort-type or cross-sectional studies; (2) evaluated MRI and/or US and/or stress radiography and/or arthrography for the diagnosis of chronic ATFL and/or CFL injury (regarding the classification of acute and chronic ligament injuries, we followed the decisions adopted by the authors of the studies included); (3) comparing imaging results with arthroscopic or surgical findings as reference standards, and (4) reported data that enabled the calculation of the number of true positive (TP), true negative (TN), false positive (FP), and false negative (FN).

The following criteria were used to exclude underqualified studies: (1) acute injury patients; (2) patients with confounding factors like ankle fracture, history of previous foot, and ankle surgeries; (3) without clearly described arthroscopic or surgical findings as their reference standards; (4) cadaveric studies or studies utilizing animal models; and (5) non-English articles.

\section{Search strategy}

A systematical literature search was conducted to include the following three databases: MEDLINE, EMBASE, and Cochrane Central Register of Controlled Trails (CENTRAL). The detailed search strategies (Table 1) were first developed in MEDLINE and were then adjusted and applied in the other two databases.

Retrieved articles from each database were at first screened for duplication. Then, after titles and abstracts screening, relevant studies for this systemic review underwent full-text screening. Eligible studies were included according to the aforementioned inclusion and exclusion criteria.

\section{Data extraction and quality assessment}

The extracted data include authors, publication years, demographic features of participants, study design, index

Table 1 Detailed search strategies

\begin{tabular}{|c|c|c|c|}
\hline Step & MEDLINE & EMBASE & CENTRAL \\
\hline 1 & $\begin{array}{l}\text { accuracy[Title/Abstract] OR accurate rate[Title/ } \\
\text { Abstract] OR diagnostic value[Title/Abstract] }\end{array}$ & $\begin{array}{l}\text { exp diagnostic accuracy/ or exp accuracy/ } \\
\text { or exp measurement accuracy/ or exp. } \\
\text { diagnostic test accuracy study/ }\end{array}$ & $\begin{array}{l}\text { MeSH descriptor: [Sensitivity and Specificity] } \\
\text { explode all trees }\end{array}$ \\
\hline 2 & sensitivity and specificity[MeSH Terms] & $\begin{array}{l}\text { sensitivity.mp.[mp=title, original title, } \\
\text { abstract, name of substance word, } \\
\text { subject heading word] }\end{array}$ & $\begin{array}{l}\text { accuracy: ti, ab, kw or accurate rate: ti, ab, kw } \\
\text { or diagnostic value: ti, ab, kw(Word variations } \\
\text { have been searched) }\end{array}$ \\
\hline 3 & $\begin{array}{l}\text { chronic ankle instability OR functional ankle } \\
\text { instability OR lateral ankle instability }\end{array}$ & $\begin{array}{l}\text { specificity.mp.[mp=title, original title, } \\
\text { abstract, name of substance word, } \\
\text { subject heading word] }\end{array}$ & $\begin{array}{l}\text { MeSH descriptor: [Lateral ligament, Ankle] } \\
\text { explode all trees }\end{array}$ \\
\hline 4 & PTFL OR CFL OR ATFL & exp ankle ligament/ & $\begin{array}{l}\text { PTFL OR CFL OR ATFL(Word variations have } \\
\text { been searched) }\end{array}$ \\
\hline 5 & $\begin{array}{l}\text { talofibular[Text Word] OR calcaneofibular[Text } \\
\text { Word] OR posterior talofibular[Text Word] OR } \\
\text { anterior talofibular[Text Word] }\end{array}$ & $\begin{array}{l}\text { ATFL.mp.[mp=title, original title, abstract, } \\
\text { name of substance word, subject heading } \\
\text { word] }\end{array}$ & $\begin{array}{l}\text { chronic ankle instability OR functional ankle } \\
\text { instability OR lateral ankle instability } \\
\text { (Word variations have been searched) }\end{array}$ \\
\hline 6 & lateral ligament, ankle[MeSH Terms] & $\begin{array}{l}\text { CFL.mp.[mp=title, original title, abstract, } \\
\text { name of substance word, subject heading } \\
\text { word] }\end{array}$ & $\begin{array}{l}\text { talofibular OR calcaneofibular OR posterior } \\
\text { talofibular OR anterior talofibular } \\
\text { (Word variations have been searched) }\end{array}$ \\
\hline 7 & (1 OR 2) AND (3 OR 4 OR 5 OR 6) & $\begin{array}{l}\text { PTFL.mp.[mp=title, original title, abstract, name } \\
\text { of substance word, subject heading word] }\end{array}$ & (1 OR 2) AND (3 OR 4 OR 5 OR 6) \\
\hline 8 & & exp ankle instability/ & \\
\hline 9 & & (1 OR 2 OR 3) AND (4 OR 5 OR 6 OR 7 OR 8) & \\
\hline
\end{tabular}


tests, gold standards, and the numbers of true positive, false negative, false positive, and true negative subjects. The pathologic features of chronic injury lead to various manifestations on images $[14,19,23]$. We eliminated this diversity by dichotomized imaging results to "injured" and "intact" in this study for better comparability among different included studies. Different kind of injuries such as "stretching," "rupture," "scarring," or "thickening" are all categorized as "injured."

Two authors independently extracted these data and filled previously drafted forms for this review. Results of the two authors were cross-validated, and discrepancies were mediated by the third author. The quality of the included articles was assessed through revised Quality Assessment of Diagnostic Accuracy Studies (QUADAS-2) tool, a widely recommended scale for diagnostic test evaluation [24]. According to QUADAS-2 tool, risk of bias was assessed in terms of patient selection, index test, and reference standard.

\section{Statistical analysis}

Sensitivity and specificity of each index test in individual study were calculated in Meta-DiSc, version 1.4.0, using the extracted data of TP, FN, FP, and TN. Pooled sensitivity and specificity were calculated using the total number of TP, FN, FP, and TN subjects in all relevant studies. Likelihood ratio (LR) evaluates the discriminatory properties of the test results [25]. Positive and negative likelihood ratio evaluates the positive and negative test results respectively. Pooled positive and negative likelihood ratio was calculated using random effects model. The diagnostic odds ratio (DOR) is defined as (true positives $\times$ true negatives) / (false positives $\times$ false negatives), which evaluates the overall diagnostic test performance combining sensitivity, specificity, and likelihood ratio [26]. Pooled DORs were calculated using random effects model.

All final outcomes were presented with 95\% confidential interval. Pooled sensitivity, specificity, likelihood ratio, and DOR are calculated concerning each subgroup. Heterogeneity testing was assessed using the $I^{2}$ test. A value of $I^{2}>50 \%$ was considered to be significant heterogeneity among the pooled data.

\section{Results}

\section{Description of included studies}

A total of 178 articles were retrieved from MEDLINE. One hundred eighty-eight articles were retrieved from EMBASE. Fifteen articles were retrieved from Cochrane Central Register of Controlled Trails. After deleting duplications, a total of 249 studies were identified in the primary search of three aforementioned databases. Then, these studies were screened for eligible studies as presented in the flow chart (Fig. 1). Twenty-four studies underwent full-text screen, and 9 studies were excluded for the following reasons: inconsistent reference standards among subjects [21, 27-29], studies on other lesions associated with chronic ankle instability [30-33], and heterogeneous population with inadequate data for chronic injury group [34].

Overall, 15 studies [35-49] were eligible for this systemic review and are summarized in Table 2. A total of 695 participants were included. In 3 studies [39, 45, 49], participants were divided into acute injury group and chronic injury one, chronic injury groups of which were included in data synthesis. Two studies $[42,44]$ reported inter-rater reliability. In one study [48], a number of ankles instead of a number of participants were considered.

\section{Methodological quality assessment}

Methodological quality assessment was conducted for each study using QUADAS-2 tool (Fig. 2). All studies mentioned that participants were diagnosed of chronic ligament injury, of which 9 studies [36-38, 42, 43, 45, $46,48,49]$ mentioned the time from initial ankle inversion trauma to study commencement or duration when patients presented with chronic symptoms. Six of 15 studies [35, 38, 39, 41, 44, 47] were categorized as high risk of bias due to patient selection. All studies mentioned arthroscopic or surgical findings as their reference standards, of which 3 studies [37, 40, 46] mentioned that the reference standard results were interpreted without knowledge of the results of the index tests. All studies mentioned that investigators were blind to arthroscopic or surgical findings when interpreting the diagnostic imaging.

\section{Diagnostic accuracy of imaging techniques}

Considering the combination of different ligaments (ATFL and CFL) and imaging techniques (MRI, US, stress radiography, and arthrography), 8 subgroups were analyzed. Extracted data of 8 subgroups are listed in Fig. 3. Pooled sensitivity, pooled specificity, and their $95 \%$ confidential interval are listed in Fig. 4 in the form of zones of mostly bad imaging efficacy (ZOMBIE) plot as Richardson [50] described. Detailed pooled data are listed in Table 3.

MRI exhibited the pooled sensitivities of $0.83[0.78,0.87]$ in diagnosing chronic ATFL injury and $0.56[0.46,0.66]$ in diagnosing chronic CFL injury. The pooled specificities were $0.88[0.82,0.93]$ in diagnosing chronic CFL injury and $0.79[0.69,0.87]$ in diagnosing chronic ATFL injury.

US presented the pooled sensitivities of $0.99[0.96,1.00]$ in diagnosing chronic ATFL injury and $0.94[0.85,0.98]$ in diagnosing CFL injury. The pooled specificities were 0.91 $[0.82,0.97]$ in diagnosing chronic ATFL injury and 0.91 [0. $80,0.97$ in diagnosing chronic CFL injury.

Stress radiography demonstrated a sensitivity of 0.81 $[0.68,0.90]$ and a specificity of $0.92[0.79,0.98]$ in diagnosing chronic ATFL injury. Two studies regarding 


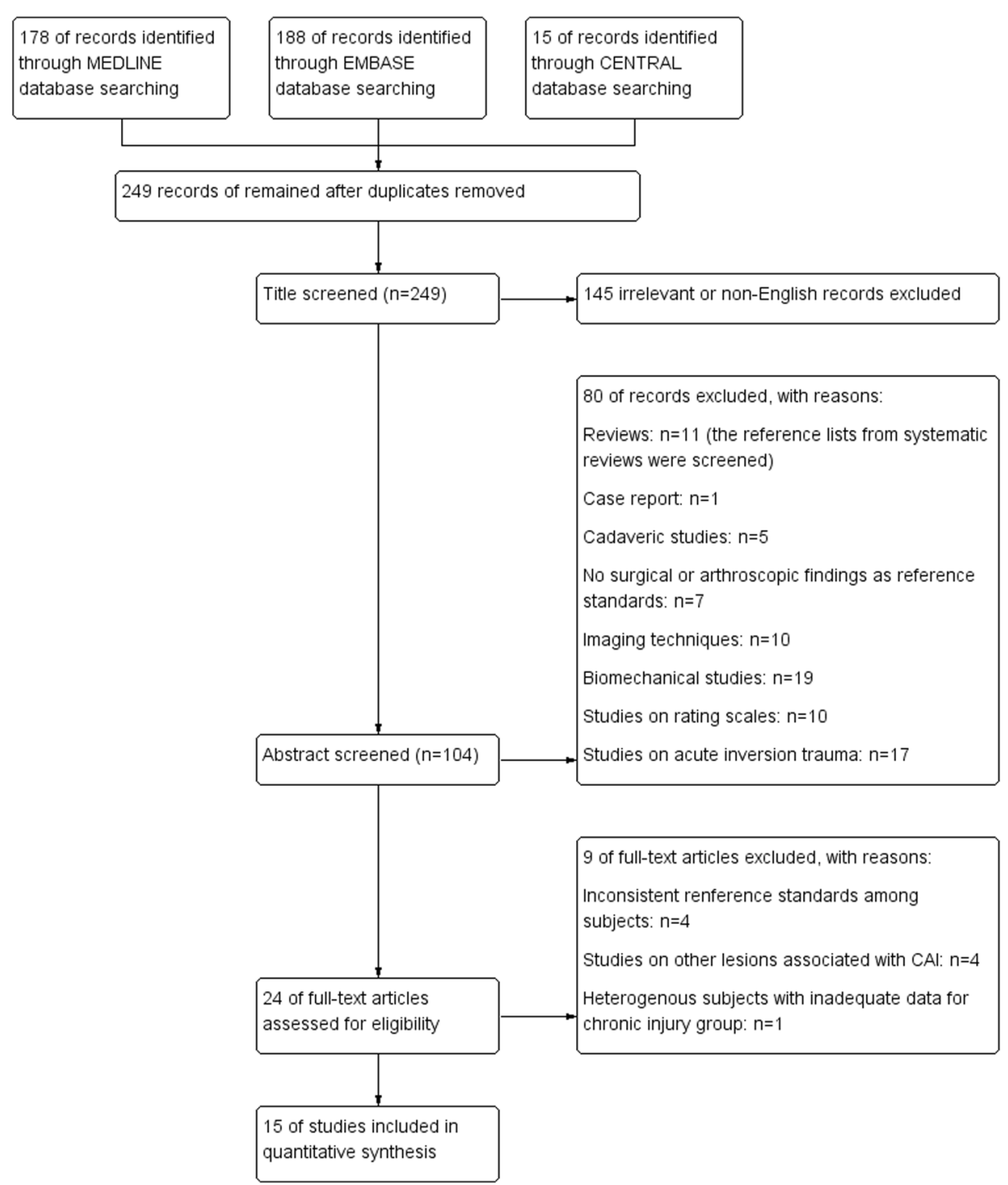

Fig. 1 Flow diagram of search strategy

stress radiography for CFL showed high heterogeneity $[43,48]$, with $I^{2}$ for pooled data above $50 \%$.

Sample size regarding arthrography in diagnosing chronic ATFL injury is relatively small, with only 34 subjects included. Arthrography presented the pooled sensitivity of 0 . $90[0.73,0.98]$ and the pooled specificity of $0.90[0.77,0.97]$ in diagnosing chronic CFL injury.

\section{Discussion}

Accurate diagnosis of chronic lateral ankle ligament injury is considered critical for surgical intervention of chronic ankle instability [13]. Imaging diagnosis is usually noninvasive and can be of value when physical tests are ambiguous [16]. Results for the four including imaging diagnostic techniques have been reported; however, the diagnostic accuracy of each imaging technique is variable in different studies, partly due to different reference standards applied [20,21]. Our study set arthroscopic or surgical findings as the gold standard to investigate four imaging techniques. This gold standard reduced the heterogeneity of different studies.

In our results, the pooled sensitivities and specificities of US in diagnosing chronic ATFL and CFL injury were all above 0.90 . When diagnosing chronic ankle instability, another systemic review involving 6 articles reported sensitivity of US ranged from 84.6 to $100 \%$ and specificity of US ranged from 90.9 to $100 \%$ [20]. In agreement with this systemic review, our results demonstrated US is an effective imaging technique in diagnosing chronic lateral ankle ligament injury.

US can precisely discriminate different ligamentous conditions, such as torn, lax, or thickened ligaments [40]. Comparing US results of healthy people and patients with chronic ankle instability, Liu et al. reported the differences 
Table 2 Summary of included studies

\begin{tabular}{|c|c|c|c|c|c|c|c|c|c|}
\hline $\begin{array}{l}\text { Study } \\
\text { (author } \\
\text { and year) }\end{array}$ & $\begin{array}{l}\text { Subject } \\
\text { features }\end{array}$ & Age & Gender & $\begin{array}{l}\text { Time from injury } \\
\text { to study } \\
\text { commencement/ } \\
\text { duration when } \\
\text { patients presented } \\
\text { with symptoms }\end{array}$ & $\begin{array}{l}\text { Study } \\
\text { design }\end{array}$ & $\begin{array}{l}\text { Level of } \\
\text { evidence }\end{array}$ & Index tests & $\begin{array}{l}\text { Gold } \\
\text { standard }\end{array}$ & Main conclusion \\
\hline $\begin{array}{l}\text { Cha et el. } \\
2012\end{array}$ & $\begin{array}{l}65 \text { patients } \\
\text { diagnosed } \\
\text { with chronic } \\
\text { lateral ankle } \\
\text { instability } \\
\text { who received } \\
\text { preoperative } \\
\text { MRI and } \\
\text { arthroscopic } \\
\text { examination }\end{array}$ & $\begin{array}{l}\text { Not } \\
\text { mentioned }\end{array}$ & $\begin{array}{l}39 \text { men } \\
\text { and } 26 \\
\text { women }\end{array}$ & Not mentioned & $\begin{array}{l}\text { Case-control } \\
\text { study }\end{array}$ & $3 b$ & MRI & $\begin{array}{l}\text { Arthroscopic } \\
\text { findings }\end{array}$ & $\begin{array}{l}\text { "However, } \\
\text { inter-observer } \\
\text { reliability and } \\
\text { sensitivity of } \\
\text { MRl findings } \\
\text { were found } \\
\text { to be relatively } \\
\text { low in this study. } \\
\text { Therefore, } \\
\text { arthroscopic } \\
\text { examination } \\
\text { before ligament } \\
\text { repair is a useful } \\
\text { and recommended } \\
\text { method for } \\
\text { diagnosis and } \\
\text { treatment of } \\
\text { intra-articular } \\
\text { lesions." }\end{array}$ \\
\hline $\begin{array}{l}\text { Chandnani et al. } \\
1994\end{array}$ & $\begin{array}{l}17 \text { chronic } \\
\text { ankle } \\
\text { instability } \\
\text { patients }\end{array}$ & $\begin{array}{l}24.5 \text { years } \\
\text { (ranged } \\
\text { from } 20 \\
\text { to } 48 \\
\text { years) }\end{array}$ & $\begin{array}{l}14 \text { men } \\
\text { and } 3 \\
\text { women }\end{array}$ & $\begin{array}{l}\text { Presented with } \\
\text { symptoms of at } \\
\text { least } 6 \text {-months } \\
\text { duration and } \\
\text { did not respond } \\
\text { to conservative } \\
\text { therapy that } \\
\text { included } \\
\text { immobilization } \\
\text { and early } \\
\text { aggressive } \\
\text { rehabilitation }\end{array}$ & Cohort study & $2 b$ & $\begin{array}{l}\text { Stress } \\
\text { radiograph, } \\
\text { MRI and MR } \\
\text { arthrography }\end{array}$ & $\begin{array}{l}\text { Findings } \\
\text { from either } \\
\text { diagnostic } \\
\text { arthroscopy } \\
(n=3) \text { or } \\
\text { open surgical } \\
\text { repair }(n=14)\end{array}$ & $\begin{array}{l}\text { "In conclusion, } \\
\text { our results } \\
\text { suggest that } \\
\text { MR arthrography } \\
\text { may be a sensitive } \\
\text { technique for } \\
\text { detecting and } \\
\text { staging tears of } \\
\text { the lateral collateral } \\
\text { ligaments and } \\
\text { evaluating } \\
\text { associated injuries } \\
\text { in patients with } \\
\text { chronic ankle } \\
\text { instability. } \\
\text { In patients for } \\
\text { whom surgery } \\
\text { is contemplated, } \\
\text { MR arthrography } \\
\text { may be a useful } \\
\text { adjunct to } \\
\text { conventional } \\
\text { imaging } \\
\text { techniques." }\end{array}$ \\
\hline $\begin{array}{l}\text { Cheng et al. } \\
2014\end{array}$ & $\begin{array}{l}120 \text { patients } \\
\text { with a clinical } \\
\text { suspicion of } \\
\text { chronic ankle } \\
\text { ligament injury }\end{array}$ & $\begin{array}{l}32 \text { years } \\
\text { (ranged from } \\
15 \text { to } 70 \text { years) }\end{array}$ & $\begin{array}{l}85 \text { men } \\
\text { and } 35 \\
\text { women }\end{array}$ & $\begin{array}{l}\text { Duration of } \\
\text { symptoms } \\
\text { ranged from } \\
6 \text { weeks to } \\
20 \text { years } \\
\text { (mean, } \\
2.2 \text { years) }\end{array}$ & $\begin{array}{l}\text { Cohort } \\
\text { study }\end{array}$ & $2 b$ & Ultrasonography & $\begin{array}{l}\text { Arthroscopic } \\
\text { findings } \\
\text { ( } 42 \text { of these } \\
\text { patients also } \\
\text { underwent } \\
\text { open surgery) }\end{array}$ & $\begin{array}{l}\text { "In conclusion, } \\
\text { ultrasonography } \\
\text { as a convenient } \\
\text { technique with } \\
\text { low costs and } \\
\text { real-time } \\
\text { examination } \\
\text { showed a } \\
\text { satisfactory } \\
\text { sensitivity and } \\
\text { specificity for } \\
\text { detecting lateral } \\
\text { ligament injury." }\end{array}$ \\
\hline $\begin{array}{l}\text { Cho et al. } \\
2016\end{array}$ & $\begin{array}{l}28 \text { consecutive } \\
\text { lateral ankle } \\
\text { instability patients } \\
\text { who underwent } \\
\text { ankle arthroscopy }\end{array}$ & $\begin{array}{l}32.4 \pm 11.9 \\
\text { years (ranged } \\
\text { from } 15 \text { to } \\
55 \text { years) }\end{array}$ & $\begin{array}{l}19 \text { men } \\
\text { and } 9 \\
\text { women }\end{array}$ & $\begin{array}{l}\text { Conservative } \\
\text { treatment had } \\
\text { failed to } \\
\text { substantially } \\
\text { alleviate the } \\
\text { symptoms for } \\
\text { at least } 3 \text { months }\end{array}$ & $\begin{array}{l}\text { Case-control } \\
\text { study }\end{array}$ & $3 b$ & $\begin{array}{l}\text { MRI, manual } \\
\text { anterior drawer } \\
\text { test, stress } \\
\text { ultrasonography, } \\
\text { and stress X-ray }\end{array}$ & $\begin{array}{l}\text { Arthroscopic } \\
\text { findings }\end{array}$ & $\begin{array}{l}\text { "Manual stress } \\
\text { US is as precise } \\
\text { as MRI in the } \\
\text { detection of } \\
\text { ligament injury } \\
\text { and provides } \\
\text { information on } \\
\text { chronic ankle } \\
\text { instability." }\end{array}$ \\
\hline $\begin{array}{l}\text { Chou et al. } \\
2006\end{array}$ & $\begin{array}{l}50 \text { patients } \\
\text { suffering from } \\
\text { ankle instability } \\
\text { (17 of them }\end{array}$ & $\begin{array}{l}\text { Ranged from } \\
14 \text { to } 77 \text { years, } \\
n=50\end{array}$ & $\begin{array}{l}29 \text { men } \\
\text { and } 21 \\
\text { women, } \\
n=50\end{array}$ & Not mentioned & $\begin{array}{l}\text { Cohort } \\
\text { study }\end{array}$ & $2 b$ & $\begin{array}{l}\text { MRI and MR } \\
\text { arthrography }\end{array}$ & $\begin{array}{l}\text { Surgical } \\
\text { findings }\end{array}$ & $\begin{array}{l}\text { "For evaluating } \\
\text { ankle disability, } \\
\text { using plain MRI } \\
\text { alone is not }\end{array}$ \\
\hline
\end{tabular}


Table 2 Summary of included studies (Continued)

\begin{tabular}{|c|c|c|c|c|c|c|c|c|c|}
\hline $\begin{array}{l}\text { Study } \\
\text { (author } \\
\text { and year) }\end{array}$ & $\begin{array}{l}\text { Subject } \\
\text { features }\end{array}$ & Age & Gender & $\begin{array}{l}\text { Time from injury } \\
\text { to study } \\
\text { commencement/ } \\
\text { duration when } \\
\text { patients presented } \\
\text { with symptoms }\end{array}$ & $\begin{array}{l}\text { Study } \\
\text { design }\end{array}$ & $\begin{array}{l}\text { Level of } \\
\text { evidence }\end{array}$ & Index tests & $\begin{array}{l}\text { Gold } \\
\text { standard }\end{array}$ & Main conclusion \\
\hline & $\begin{array}{l}\text { who would } \\
\text { receive surgical } \\
\text { intervention } \\
\text { were included } \\
\text { in this review.) }\end{array}$ & & & & & & & & $\begin{array}{l}\text { adequate for } \\
\text { correctly detecting } \\
\text { lateral collateral } \\
\text { ligamentous injury } \\
\text { of the ankle joint. } \\
\text { MR arthrography } \\
\text { improves the } \\
\text { sensitivity and the } \\
\text { accuracy for ATaF } \\
\text { and CF ligament } \\
\text { injuries. It also } \\
\text { helps in assessing } \\
\text { coexisting } \\
\text { pathologic lesions } \\
\text { of ankle joints." }\end{array}$ \\
\hline $\begin{array}{l}\text { Hua et al. } \\
2012\end{array}$ & $\begin{array}{l}83 \text { consecutive } \\
\text { patients } \\
\text { underwent } \\
\text { ankle arthroscopy } \\
\text { for different } \\
\text { diagnosis }\end{array}$ & $\begin{array}{l}32.2 \text { years } \\
\text { (ranged from } \\
17 \text { to } 57 \text { years) }\end{array}$ & $\begin{array}{l}51 \text { men } \\
\text { and } 32 \\
\text { women }\end{array}$ & Not mentioned & $\begin{array}{l}\text { Case-control } \\
\text { study }\end{array}$ & $3 b$ & Ultrasonography & $\begin{array}{l}\text { Arthroscopic } \\
\text { findings }\end{array}$ & $\begin{array}{l}\text { "US examination } \\
\text { is a reliable and } \\
\text { accurate method } \\
\text { to evaluate chronic } \\
\text { ATFL injury." }\end{array}$ \\
\hline $\begin{array}{l}\text { Joshy et al. } \\
2010\end{array}$ & $\begin{array}{l}24 \text { patients } \\
\text { underwent } \\
\text { arthroscopy as } \\
\text { well as MRI for } \\
\text { chronic ankle } \\
\text { pain and/or } \\
\text { instability }\end{array}$ & $\begin{array}{l}39 \text { years } \\
\text { (range from } \\
11 \text { to } 65 \text { years) }\end{array}$ & $\begin{array}{l}12 \text { men } \\
\text { and } 12 \\
\text { women }\end{array}$ & Not mentioned & $\begin{array}{l}\text { Case-control } \\
\text { study }\end{array}$ & $3 b$ & MRI & $\begin{array}{l}\text { Arthroscopic } \\
\text { findings }\end{array}$ & $\begin{array}{l}\text { "MRI scan has very } \\
\text { high specificity and } \\
\text { positive predictive } \\
\text { value in diagnosing } \\
\text { tears of ATFT, CFL } \\
\text { and osteochondral } \\
\text { lesions. However } \\
\text { sensitivity and } \\
\text { negative predictive } \\
\text { value is low with } \\
\text { MRI." }\end{array}$ \\
\hline $\begin{array}{l}\text { Kumar et al. } \\
2007\end{array}$ & $\begin{array}{l}58 \text { patients with } \\
\text { symptomatic } \\
\text { instability of } \\
\text { their ankle }\end{array}$ & $\begin{array}{l}28 \text { years } \\
\text { (ranged from } \\
18 \text { to } 50 \text { years) }\end{array}$ & $\begin{array}{l}47 \text { men } \\
\text { and } 11 \\
\text { women }\end{array}$ & $\begin{array}{l}\text { Unresponsive } \\
\text { to physiotherapy } \\
\text { and non-operative } \\
\text { management for } \\
\text { at least } 6 \text { months }\end{array}$ & $\begin{array}{l}\text { Cohort } \\
\text { study }\end{array}$ & $2 b$ & $\begin{array}{l}\text { MRI and stress } \\
\text { radiography }\end{array}$ & $\begin{array}{l}\text { Arthroscopic } \\
\text { findings }\end{array}$ & $\begin{array}{l}\text { "MRI did not } \\
\text { demonstrate } \\
\text { any distinct } \\
\text { advantage over } \\
\text { the examination } \\
\text { under anesthesia } \\
\text { and stress } \\
\text { radiography in } \\
\text { the diagnosis } \\
\text { of grade III lateral } \\
\text { ankle } \\
\text { ligament } \\
\text { injuries." }\end{array}$ \\
\hline $\begin{array}{l}\text { Lee et al. } \\
2012\end{array}$ & $\begin{array}{l}34 \text { patients who } \\
\text { underwent ankle } \\
\text { MRI because of } \\
\text { ankle sprains or } \\
\text { ankle instability }\end{array}$ & $\begin{array}{l}29 \text { years } \\
\text { (ranged from } \\
13 \text { to } 53 \text { years) }\end{array}$ & $\begin{array}{l}22 \text { men } \\
\text { and } 12 \\
\text { women }\end{array}$ & Not mentioned & $\begin{array}{l}\text { Cohort } \\
\text { study }\end{array}$ & $2 b$ & MRI & $\begin{array}{l}\text { Arthroscopic } \\
\text { findings }\end{array}$ & $\begin{array}{l}\text { "|A cortical } \\
\text { defect with } \\
\text { a bright } \\
\text { dot-like or } \\
\text { curvilinear } \\
\text { high-signal- } \\
\text { intensity } \\
\text { lesion on } \\
\text { T2-weighted } \\
\text { MRI may be } \\
\text { regarded as } \\
\text { an additional } \\
\text { finding to } \\
\text { help increase }\end{array}$ \\
\hline
\end{tabular}


Table 2 Summary of included studies (Continued)

\begin{tabular}{|c|c|c|c|c|c|c|c|c|c|}
\hline $\begin{array}{l}\text { Study } \\
\text { (author } \\
\text { and year) }\end{array}$ & $\begin{array}{l}\text { Subject } \\
\text { features }\end{array}$ & Age & Gender & $\begin{array}{l}\text { Time from injury } \\
\text { to study } \\
\text { commencement/ } \\
\text { duration when } \\
\text { patients presented } \\
\text { with symptoms }\end{array}$ & $\begin{array}{l}\text { Study } \\
\text { design }\end{array}$ & $\begin{array}{l}\text { Level of } \\
\text { evidence }\end{array}$ & Index tests & $\begin{array}{l}\text { Gold } \\
\text { standard }\end{array}$ & Main conclusion \\
\hline & & & & & & & & & $\begin{array}{l}\text { the diagnostic } \\
\text { performance } \\
\text { for detecting } \\
\text { anterior } \\
\text { talofibular } \\
\text { ligament } \\
\text { injuries, } \\
\text { including } \\
\text { partial tears." }\end{array}$ \\
\hline $\begin{array}{l}\text { Oae et al. } \\
2010\end{array}$ & $\begin{array}{l}34 \text { consecutive } \\
\text { patients needed } \\
\text { an operation } \\
\text { because of } \\
\text { severe problems } \\
\text { such as } \\
\text { osteochondral } \\
\text { lesions, synovitis } \\
\text { and instability } \\
\text { (15 chronic } \\
\text { cases of them } \\
\text { were included } \\
\text { in this review.) }\end{array}$ & $\begin{array}{l}29 \text { years } \\
\text { (ranged } \\
\text { from } 13 \\
\text { to } 55 \text { years), } \\
n=34\end{array}$ & $\begin{array}{l}19 \text { men } \\
\text { and } 15 \\
\text { women, } \\
n=34\end{array}$ & $\begin{array}{l}\text { With a history } \\
\text { of ankle injury } \\
\text { of more than } \\
4 \text { weeks }\end{array}$ & $\begin{array}{l}\text { Cohort } \\
\text { study }\end{array}$ & $2 b$ & $\begin{array}{l}\text { Stress } \\
\text { radiography, } \\
\text { ultrasonography, } \\
\text { and MRI }\end{array}$ & $\begin{array}{l}\text { Arthroscopic } \\
\text { findings }\end{array}$ & $\begin{array}{l}\text { "We obtained } \\
\text { satisfactory } \\
\text { results with } \\
\text { US and MR } \\
\text { imaging." }\end{array}$ \\
\hline $\begin{array}{l}\text { Park et al. } \\
2012\end{array}$ & $\begin{array}{l}48 \text { people } \\
\text { suspected of } \\
\text { chronic ankle } \\
\text { ligament injury }\end{array}$ & $\begin{array}{l}36 \text { years } \\
\text { (ranged from } \\
19 \text { to } 64 \text { years) }\end{array}$ & $\begin{array}{l}25 \text { men } \\
\text { and } 23 \\
\text { women }\end{array}$ & $\begin{array}{l}\text { The duration } \\
\text { of symptoms } \\
\text { ranging from } \\
\text { several months } \\
\text { to several years }\end{array}$ & $\begin{array}{l}\text { Cohort } \\
\text { study }\end{array}$ & $2 b$ & MRI & $\begin{array}{l}\text { Surgical } \\
\text { findings }\end{array}$ & $\begin{array}{l}\text { "In conclusion, } \\
\text { MRI does not } \\
\text { show perfect } \\
\text { sensitivity for } \\
\text { the evaluation } \\
\text { of chronic lateral } \\
\text { ankle ligament } \\
\text { injury, such as } \\
\text { those to the ATFL } \\
\text { and CFL. Diagnosis } \\
\text { of a complete tear } \\
\text { of the ATFL on MRI } \\
\text { is more sensitive } \\
\text { than the diagnosis } \\
\text { of a complete tear } \\
\text { of the CFL. MRI } \\
\text { findings of CFL } \\
\text { injury were } \\
\text { diagnostically } \\
\text { specific but } \\
\text { were not sensitive." }\end{array}$ \\
\hline $\begin{array}{l}\text { Staats et al. } \\
2017\end{array}$ & $\begin{array}{l}30 \text { patients with } \\
\text { CAl and failed } \\
\text { conservative } \\
\text { treatment }\end{array}$ & $\begin{array}{l}39.1 \text { years } \\
\text { (ranged from } \\
18 \text { to } 71 \text { years) }\end{array}$ & $\begin{array}{l}15 \text { men } \\
\text { and } 15 \\
\text { women }\end{array}$ & Not mentioned & $\begin{array}{l}\text { Cohort } \\
\text { study }\end{array}$ & $2 b$ & $M R I$ & $\begin{array}{l}\text { Arthroscopic } \\
\text { findings }\end{array}$ & $\begin{array}{l}\text { "MRI is a helpful } \\
\text { tool for preoperative } \\
\text { evaluation, but } \\
\text { arthroscopy remains } \\
\text { gold standard in the } \\
\text { diagnosis of } \\
\text { associated lesions } \\
\text { in patients with CAl" }\end{array}$ \\
\hline $\begin{array}{l}\text { Sugimoto } \\
\text { et al. } 2002\end{array}$ & $\begin{array}{l}37 \mathrm{ft} \text { of } 35 \\
\text { patients in } \\
\text { whom recurrent } \\
\text { instability of } \\
\text { the ankle was } \\
\text { diagnosed }\end{array}$ & $\begin{array}{l}29.1 \text { years } \\
\text { (ranged from } \\
11 \text { to } 56 \text { years) }\end{array}$ & $\begin{array}{l}16 \text { men } \\
\text { and } 19 \\
\text { women }\end{array}$ & $\begin{array}{l}\text { Mean interval } \\
\text { between the } \\
\text { injury and } \\
\text { arthrography } \\
\text { was } 4 \text { years } \\
3 \text { months } \\
\text { (range, } \\
6 \text { months- } \\
22 \text { years) }\end{array}$ & $\begin{array}{l}\text { Cohort } \\
\text { study }\end{array}$ & $2 b$ & $\begin{array}{l}\text { Subtalar } \\
\text { arthrography, } \\
\text { manual anterior } \\
\text { drawer test and } \\
\text { talar tilt test }\end{array}$ & $\begin{array}{l}\text { Surgical } \\
\text { findings }\end{array}$ & $\begin{array}{l}\text { "Subtalar } \\
\text { arthrography } \\
\text { is valuable in } \\
\text { making the } \\
\text { diagnosis of } \\
\text { calcaneofibular } \\
\text { ligament injury } \\
\text { in recurrent } \\
\text { instability } \\
\text { of the ankle." }\end{array}$ \\
\hline $\begin{array}{l}\text { Tan et al. } \\
2017\end{array}$ & $\begin{array}{l}82 \text { patients with } \\
\text { ankle inversion } \\
\text { trauma history } \\
\text { (42 in the chronic } \\
\text { group were } \\
\text { included in } \\
\text { this review.) }\end{array}$ & $\begin{array}{l}24.8 \text { years } \\
\text { (ranged } \\
\text { from } 17 \text { to } \\
48 \text { years), } \\
n=42\end{array}$ & $\begin{array}{l}36 \text { men } \\
\text { and } 6 \\
\text { women, } \\
n=42\end{array}$ & $\begin{array}{l}\text { Interval from } \\
\text { time of injury } \\
\text { to time of MRI } \\
\text { more than } \\
3 \text { months }\end{array}$ & $\begin{array}{l}\text { Cohort } \\
\text { study }\end{array}$ & $2 b$ & MRI & $\begin{array}{l}\text { Surgical } \\
\text { findings }\end{array}$ & $\begin{array}{l}\text { "MRI was able } \\
\text { to accurately } \\
\text { diagnose } \\
\text { lateral ankle } \\
\text { ligament tears } \\
\text { in most cases. } \\
\text { Diagnosis of a } \\
\text { complete ATFL }\end{array}$ \\
\hline
\end{tabular}


Table 2 Summary of included studies (Continued)

\begin{tabular}{|c|c|c|c|c|c|c|c|c|c|}
\hline $\begin{array}{l}\text { Study } \\
\text { (author } \\
\text { and year) }\end{array}$ & $\begin{array}{l}\text { Subject } \\
\text { features }\end{array}$ & Age & Gender & $\begin{array}{l}\text { Time from injury } \\
\text { to study } \\
\text { commencement/ } \\
\text { duration when } \\
\text { patients presented } \\
\text { with symptoms }\end{array}$ & $\begin{array}{l}\text { Study } \\
\text { design }\end{array}$ & $\begin{array}{l}\text { Level of } \\
\text { evidence }\end{array}$ & Index tests & $\begin{array}{l}\text { Gold } \\
\text { standard }\end{array}$ & Main conclusion \\
\hline & & & & & & & & & $\begin{array}{l}\text { tear on MRI is } \\
\text { more sensitive } \\
\text { than that of } \\
\text { complete CFL } \\
\text { tear. The MRI } \\
\text { findings of } \\
\text { CFL injury were } \\
\text { diagnostically } \\
\text { specific but } \\
\text { were not } \\
\text { sensitive." }\end{array}$ \\
\hline
\end{tabular}

in the thickness of ATFL [51] and Croy et al. reported differences in the length of ATFL [52]. Lee et al. recommended stress US over regular one for diagnosis of chronic lateral ankle ligament injury [53]. The study of Cho et al., which was included in the current review [38], utilized stress US. Other studies included in the current review did not utilize stress US technique, but still presented high diagnostic accuracy. However, US is of limited value in assessing bone or cartilage and highly experiencereliant and may be much less efficacious in less experienced hands. Moreover, only 4 studies [37, 38, 40, 45] included in this systemic review were related to the US examination of chronic lateral ankle ligament injuries, of them only one study [37] involved CFL injuries. Only two included studies $[38,45]$ compared US with MRI findings and reported similar diagnostic effectiveness. US is an effective imaging technique in diagnosing chronic lateral ankle ligament injury, yet more studies are warranted to compare US with other imaging techniques.

In our results, MRI exhibited the pooled sensitivities of 0.83 in diagnosing chronic ATFL injury and 0.56 in diagnosing chronic CFL injury. The pooled specificities were around 0.8 in diagnosing chronic ATFL and CFL injury. In clinical practice, MRI is highly recommended in diagnosing ligamentous injuries [54]. Also, MRI was reported to be effective in diagnosing intra-articular lesions of chronic ankle instability, including osteochondral lesions of talus, syndesmotic injuries, and impingement syndromes [55, 56]. Using MRI, Tao et al. [57] reported more cartilage lesions in patients with combined injuries of the ATFL and CFL, compared to patients with only ATFL injury. A study showed that $86.7 \%$ of the experts recommended MRI before considering surgery in chronic ankle instability patients [18]. However, according to our results, MRI did not provide the highest sensitivity or specificity in diagnosing chronic ligament injuries. It presented different patterns in diagnosing chronic ATFL and CFL injuries. The sensitivity for diagnosing chronic ATFL injury $(0.83[0.78,0.87])$ was higher than that for diagnosing chronic CFL injury $(0.56[0.46,0.66])$. MRI is still irreplaceable in assessing chronic lateral ankle ligament injury because it is frequently performed to confirm or exclude the presence of concomitant lesions and influence the precise surgical technique for a certain patient. Two included studies $[38,45]$ in the current systemic review compared US with MRI findings and did not report significantly different diagnostic effectiveness.

Arthroscopy was recommended as a complementary to MRI for definitive diagnosis [17, 18, 22]. An expert consensus from Guillo et al. recommended that an arthroscopy should be performed at the time of surgery unless intra-articular pathology has been excluded by MRI scan and there is no history of pain [17].

In our results, stress radiography demonstrated a sensitivity of 0.81 and a specificity of 0.92 in diagnosing chronic ATFL injury. Tourné et al. suggested that stress radiography presented high specificity (up to $100 \%$ ) but low sensitivity (57\%), suggesting dynamic radiographs only have diagnostic value if they are positive [58]. According to our results, stress radiography showed similar high specificity as US. When diagnosing chronic ATFL injury, the sensitivity of stress radiography is still above 0.80 , and the pooled LRis 0.24 [0.11-0.54], similar as that of MRI. Negative stress radiographic findings decreases the post-test probability of chronic ATFL and CFL injury in patients with ankle inversion trauma history. However, ligamentous laxity detected through stress radiography is not synonymous with chronic ankle instability [59]. Large variability in talar tilt and anterior drawer stress radiography precludes their routine use in diagnosing chronic ankle instability [60].

The role of anesthesia in stress radiography was reported by McCaskie et al. [61], suggesting larger discriminative capability under anesthesia. Amongst studies included in the current review, only one study reported stress radiography under anesthesia [43]. The large heterogeneity between stress radiography results for chronic CFL injury may partly explained by anesthetic condition. Though stress radiography is helpful in the diagnosis, it is hardly a useful tool in predicting surgical outcomes. Jeong et al. reported stress radiography-positive patients were not statistically 


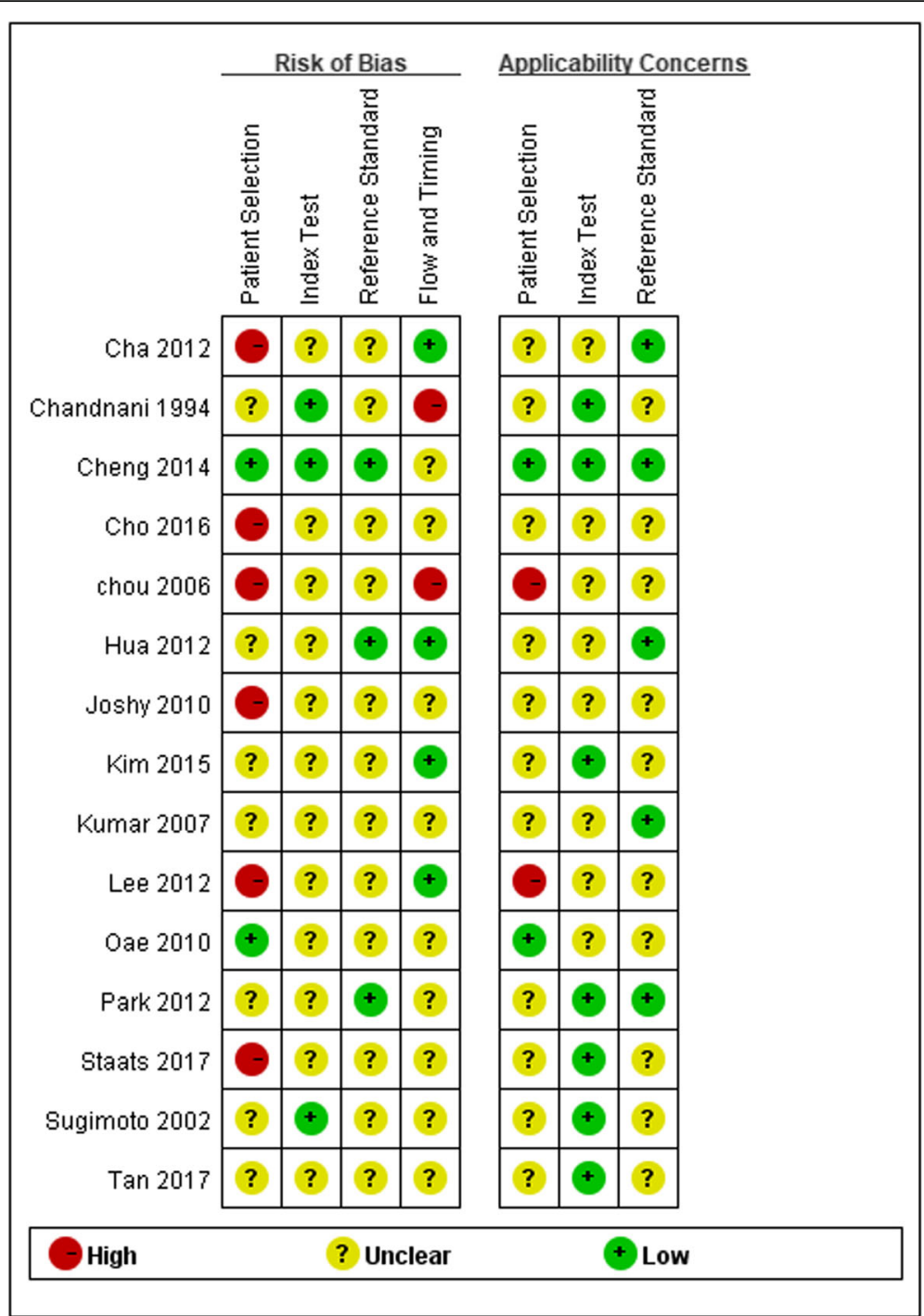

Fig. 2 Methodological quality assessment of included studies using QUADAS-2 tool. Red stands for high risk. Green stands for low risk. Yellow stands for unclear risk

significantly different from stress radiography-negative patients in prognostic features [62].

Arthrography is indicated for staging and detecting intra-articular lesions of chronic lateral ankle instability [63]. Samoto et al. reported that patients with combined injuries of the ATFL and CFL diagnosed through arthrography had worse prognosis than patients with only ATFL injury [64]. Arthrography is an invasive imaging technique. With the spread of arthroscopy, which is also invasive but much more accurate, this technique is now losing its popularity. Only 3 studies before year 2010 discussed arthrography $[36,39,48]$.

There are several limitations in the current systemic review. First, 6 of the 15 included studies were graded as high risk of bias due to patient selection. Unlike metaanalysis of clinical intervention, in meta-analysis of diagnostic tests, it is common to include case-control studies considered as high risk of bias [65]. Case-control studies create a preselected patient population and should be interpreted with caution. Second, associated lesions of chronic lateral ankle ligament injury were not discussed in the current review; however, these associated lesions spotted on images would certainly affect the judgement of clinicians. Third, some studies compared the diagnostic accuracy of identical imaging technique with different parameters and/or configurations on diagnosing chronic lateral ankle ligament injury. Strength of the MRI machines varied among different studies. This diversity in configuration may cause the heterogeneity within each subgroup in this study. Moreover, the size of the included studies was relatively small. Of the 15 included studies, only a total of 695 participants were 


\section{a MRI-ATFL}

$\begin{array}{lrrrrr}\text { Study } & \text { TP } & \text { FP } & \text { FN } & \text { TN } & \text { Sensitivity }(95 \% \text { Cl) } \\ \text { Cha 2012 } & 39 & 0 & 26 & 0 & 0.60[0.47,0.72] \\ \text { Chandnani 1994 } & 7 & 0 & 7 & 3 & 0.50[0.23,0.77] \\ \text { Cho 2016 } & 28 & 0 & 0 & 0 & 1.00[0.88,1.00] \\ \text { chou 2006 } & 12 & 0 & 2 & 3 & 0.86[0.57,0.98] \\ \text { Joshy 2010 } & 4 & 0 & 2 & 18 & 0.67[0.22,0.96] \\ \text { Kim 2015 } & 44 & 3 & 11 & 21 & 0.80[0.67,0.90] \\ \text { Kumar 2007 } & 22 & 13 & 3 & 20 & 0.88[0.69,0.97] \\ \text { Lee 2012 } & 31 & 1 & 2 & 0 & 0.94[0.80,0.99] \\ \text { Oae 2010 } & 12 & 0 & 1 & 2 & 0.92[0.64,1.00] \\ \text { Park 2012 } & 39 & 1 & 5 & 3 & 0.89[0.75,0.96] \\ \text { Staats 2017 } & 30 & 0 & 0 & 0 & 1.00[0.88,1.00] \\ \text { Tan 2017 } & 32 & 1 & 9 & 0 & 0.78[0.62,0.89]\end{array}$

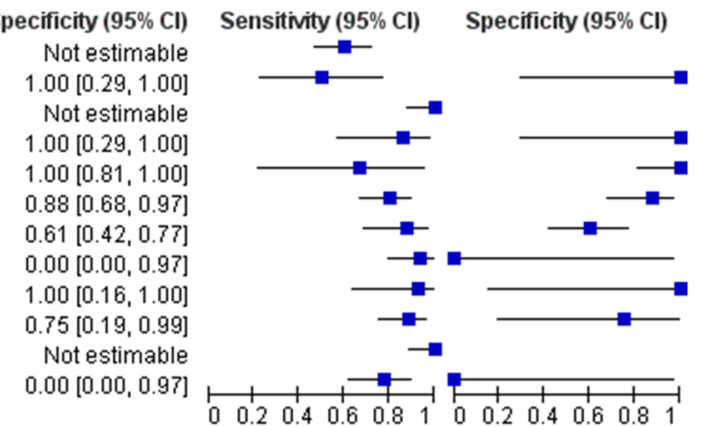

Arthrography-ATFL

Study TP FP FN TN Sensitivity $(95 \% \mathrm{Cl})$ Specificity $(95 \% \mathrm{Cl})$

$\begin{array}{lllllll}\text { Chandnani } 1994 & 14 & 0 & 0 & 3 & 1.00[0.77,1.00] & 1.00[0.29,1.00]\end{array}$

$\begin{array}{lllllll}\text { chou } 2006 & 14 & 0 & 0 & 3 & 1.00[0.77,1.00] & 1.00[0.29,1.00]\end{array}$

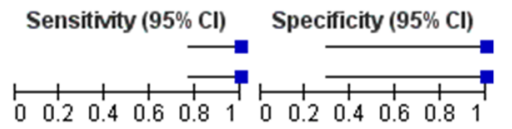

stress radiography-ATFL

\begin{tabular}{|c|c|c|c|c|c|c|c|}
\hline \multicolumn{2}{|l|}{ Study } & TP & FP & $\mathrm{FN}$ & TN & Sensitivity $(95 \% \mathrm{Cl})$ & Specificity (95\% \\
\hline \multicolumn{2}{|c|}{ Chandnani 1994} & 9 & 0 & 5 & 3 & $0.64[0.35,0.87]$ & $1.00[0.29,1$. \\
\hline \multicolumn{2}{|l|}{ Cho 2016} & 24 & 0 & 4 & 0 & $0.86[0.67,0.96]$ & Not estir \\
\hline \multicolumn{2}{|l|}{ Kumar 2007} & 21 & 3 & 4 & 30 & $0.84[0.64,0.95]$ & $0.91[0.7$ \\
\hline \multicolumn{2}{|l|}{ Oae 2010} & 12 & 0 & 1 & 2 & $0.92[0.64,1.00]$ & $1.00[0$ \\
\hline \multicolumn{8}{|c|}{ ultrasound-ATFL } \\
\hline Study & TP & FP & $\mathrm{FN}$ & TN & \multicolumn{2}{|c|}{ Sensitivity $(95 \% \mathrm{Cl}) \mathrm{S}$} & Specificity $(95 \%$ Cl) \\
\hline Cheng 2014 & 93 & 1 & 1 & 25 & \multirow{2}{*}{\multicolumn{2}{|c|}{$\begin{array}{l}0.99[0.94,1.00] \\
1.00[0.88,1.00]\end{array}$}} & $0.96[0.80,1.00]$ \\
\hline Cho 2016 & 28 & 0 & 0 & 0 & & & \multirow{2}{*}{ Not estimable } \\
\hline Hua 2012 & 43 & 3 & 1 & 36 & \multicolumn{2}{|r|}{$\begin{array}{l}1.00[0.88,1.00] \\
0.98[0.88,1.00]\end{array}$} & \\
\hline Oae 2010 & 13 & 2 & 0 & 0 & \multicolumn{2}{|r|}{$1.00[0.75,1.00]$} & $0.00[0.00,0.84]$ \\
\hline
\end{tabular}

b

MRI-CFL

$\begin{array}{lrrrrrr}\text { Study } & \text { TP } & \text { FP } & \text { FN } & \text { TN } & \text { Sensitivity }(95 \% \text { Cl) } & \text { Specificity }(95 \% \text { Cl }) \\ \text { Chandnani 1994 } & 5 & 1 & 5 & 5 & 0.50[0.19,0.81] & 0.83[0.36,1.00 \\ \text { chou 2006 } & 2 & 2 & 4 & 9 & 0.33[0.04,0.78] & 0.82[0.48,0.98] \\ \text { Joshy 2010 } & 9 & 7 & 10 & 32 & 0.47[0.24,0.71] & 0.82[0.66,0.92] \\ \text { Kumar 2007 } & 8 & 7 & 10 & 33 & 0.44[0.22,0.69] & 0.82[0.67,0.93 \\ \text { Park 2012 } & 15 & 1 & 4 & 28 & 0.79[0.54,0.94] & 0.97[0.82,1.00 \\ \text { Staats 2017 } & 4 & 0 & 1 & 25 & 0.80[0.28,0.99] & 1.00[0.86,1.00 \\ \text { Tan 2017 } & 15 & 2 & 11 & 14 & 0.58[0.37,0.77] & 0.88[0.62,0.98\end{array}$

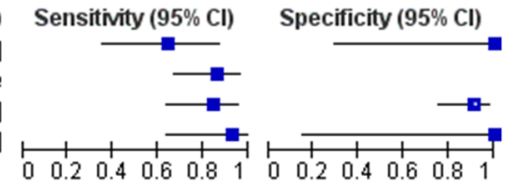

Arthrography-CFL
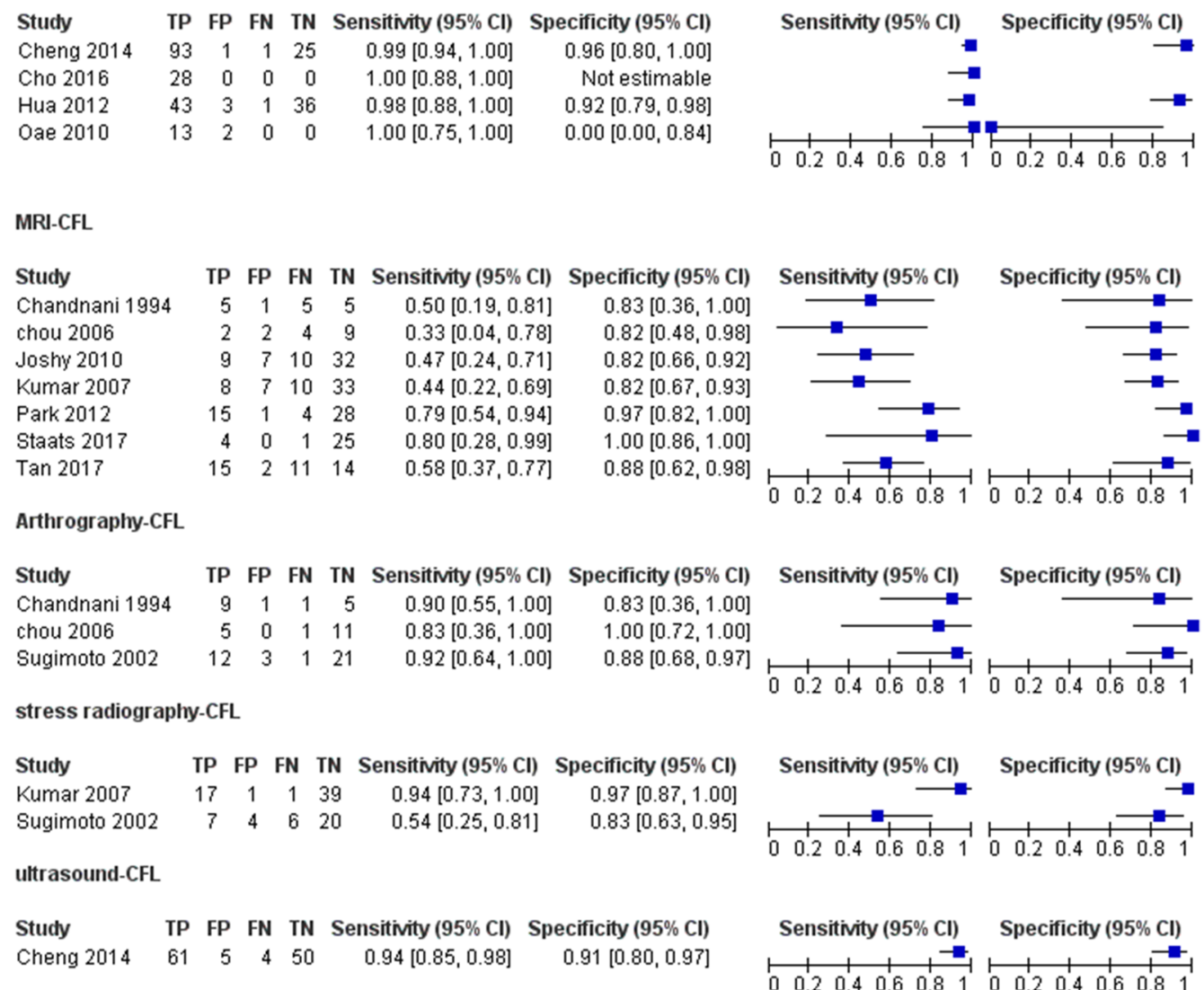

Fig. 3 Forest plot showing sensitivity and specificity for each included study. MRI, US, stress radiography, and arthrography studies for ATFL and CFL are listed in $\mathbf{a}$ and $\mathbf{b}$. TP, true positive; FP, false positive; FN, false negative; TN, true negative 

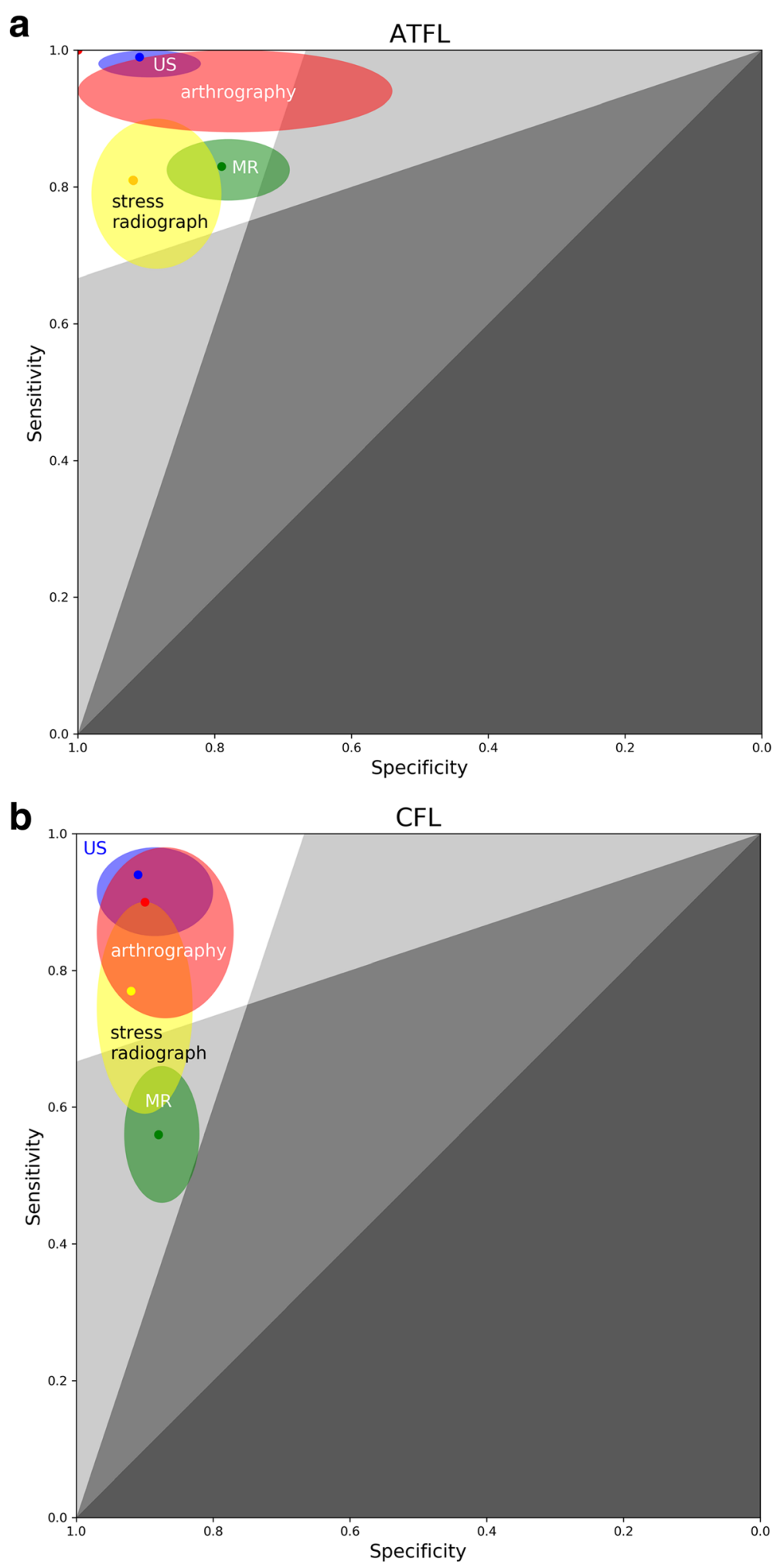

Fig. 4 Zones of mostly bad imaging efficacy (ZOMBIE) plot for ATFL (a) and CFL (b). This is based on the receiver operating characteristic (ROC) plot. The colored dot stands for pooled sensitivity and specificity of each imaging technique, and the colored oval stands for $95 \%$ confidence interval of each imaging technique (green, MR; blue, US; red, arthrography; yellow, stress radiograph). Upper-left boomerang-shaped area formed by white and light grey zones defines zone of acceptable efficacy. Upper arm of the boomerang-shaped area stands for negative likelihood ratio below 0.33. Left arm of the boomerang-shaped area stands for positive likelihood ratio above 3 


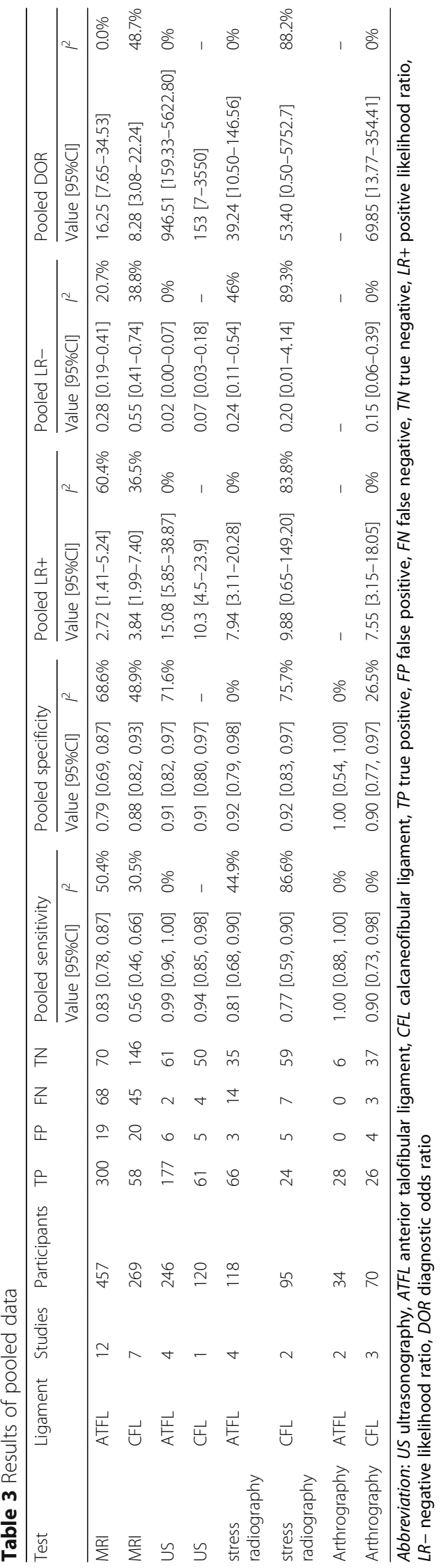


included. Studies reporting the imaging diagnosis of PTFL injury are not sufficient to draw meaningful conclusion. The studies comparing different imaging techniques for the same group of subjects are limited. This suggests more diagnostic studies with high quality are warranted for imaging diagnosing chronic lateral ankle ligament injury.

\section{Conclusion}

This systematic review with meta-analysis investigated the accuracy of imaging for the diagnosis of chronic lateral ankle ligament injury. Our results demonstrated that ultrasound manifested high diagnostic accuracy in diagnosing chronic lateral ankle ligament injury. MRI presented the diagnostic sensitivity and specificity around 0.8 for chronic ATFL injuries, but much lower sensitivity for chronic CFL injuries. Clinicians should be aware of the limitations of MRI in detecting chronic CFL injuries. Stress radiography showed similar high specificity as US in diagnosing chronic lateral ankle ligament injury and showed diagnostic value of both positive and negative results. Arthrography demonstrated sensitivity and specificity around 0.9 in diagnosing chronic CFL injury, but limited literature recommended arthrography due to invasiveness, especially in recent years. When the diagnosis of chronic lateral ankle ligament injury is uncertain after careful inquiry of the patient's history and physical tests, US may be a helpful imaging technique in experienced hands.

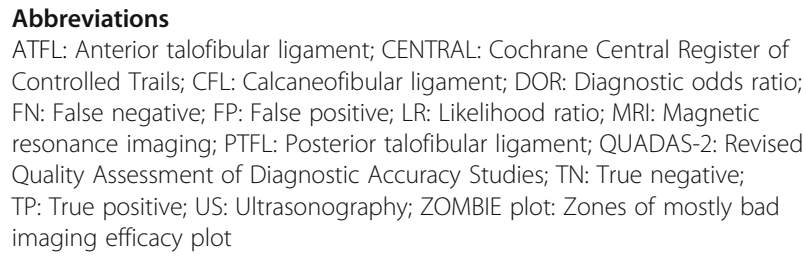

ATFL: Anterior talofibular ligament; CENTRAL: Cochrane Central Register of Controlled Trails; CFL: Calcaneofibular ligament; DOR: Diagnostic odds ratio; FN: False negative; FP: False positive; LR: Likelihood ratio; MRI: Magnetic resonance imaging; PTFL: Posterior talofibular ligament; QUADAS-2: Revised Quality Assessment of Diagnostic Accuracy Studies; TN: True negative; TP: True positive; US: Ultrasonography; ZOMBIE plot: Zones of mostly bad imaging efficacy plot

\section{Funding}

Dr. Xin Ma is currently receiving grants from National Natural Science Foundation of China (Grant No. 81171670 and Grant No. 81472037). Dr. Chen Wang is currently receiving grants from National Natural Science Foundation of China (Grant No. 81601862).

\section{Availability of data and materials}

All data generated or analyzed during this study are included in this published article.

\section{Authors' contributions}

All authors were fully involved in the study and preparation of the manuscript. SC and CW contributed to the study design and preparation of the manuscript. XM contributed to the search strategies. XW contributed to the statistical analysis. JH and $\mathrm{CZ}$ contributed to the article screening and data extraction. All authors read and approved the final manuscript.

\section{Competing interests}

The authors declare that they have no competing interests.

\section{Publisher's Note}

Springer Nature remains neutral with regard to jurisdictional claims in published maps and institutional affiliations.
Received: 19 February 2018 Accepted: 11 April 2018

Published online: 22 May 2018

\section{References}

1. Doherty C, Delahunt E, Caulfield B, Hertel J, Ryan J, Bleakley C. The incidence and prevalence of ankle sprain injury: a systematic review and meta-analysis of prospective epidemiological studies. Sports Med. 2014;44(1):123-40.

2. Fong DT, Hong Y, Chan LK, Yung PS, Chan KM. A systematic review on ankle injury and ankle sprain in sports. Sports Med. 2007;37(1):73-94.

3. Waterman BR, Owens BD, Davey S, Zacchilli MA, Belmont PJ Jr. The epidemiology of ankle sprains in the United States. J Bone Joint Surg Am. 2010;92(13):2279-84.

4. Bonnel F, Toullec E, Mabit C, Tourne Y. Chronic ankle instability: biomechanics and pathomechanics of ligaments injury and associated lesions. Orthop Traumatol Surg Res. 2010;96(4):424-32.

5. Hertel J. Functional anatomy, pathomechanics, and pathophysiology of lateral ankle instability. J Athl Train. 2002;37(4):364-75.

6. Delahunt E, Coughlan GF, Caulfield B, Nightingale EJ, Lin CWC, Hiller CE. Inclusion criteria when investigating insufficiencies in chronic ankle instability. Med Sci Sports Exerc. 2010;42(11):2106-21.

7. de Vries JS, Krips R, Sierevelt IN, Blankevoort L, van Dijk CN. Interventions for treating chronic ankle instability. Cochrane Database Syst Rev. 2011; 10(8): Cd004124.

8. Mailuhu AKE, Oei EHG, van Putte-Katier N, van Ochten JM, Bindels PJE, Bierma-Zeinstra SMA, van Middelkoop M. Clinical and radiological predictors for persistent complaints five years after a lateral ankle sprain: a long-term follow-up study in primary care. J Sci Med Sport. 2017; 21(3):250-6.

9. Pourkazemi F, Hiller CE, Raymond J, Nightingale EJ, Refshauge KM. Predictors of chronic ankle instability after an index lateral ankle sprain: a systematic review. J Sci Med Sport. 2014;17(6):568-73.

10. Vega J, Pena F, Golano P. Minor or occult ankle instability as a cause of anterolateral pain after ankle sprain. Knee Surg Sports Traumatol Arthrosc. 2016;24(4):1116-23.

11. Hubbard TJ, Hertel J, Olmsted-Kramer L, Denegar CR. Contributing factors to chronic ankle instability. Med Sci Sports Exerc. 2006;38(5):S86-7.

12. Kerr HL, Bayley E, Jackson R, Kothari P. The role of arthroscopy in the treatment of functional instability of the ankle. Foot Ankle Surg. 2013;19(4):273-5.

13. Rodriguez-Merchan EC. Chronic ankle instability: diagnosis and treatment. Arch Orthop Trauma Sur. 2012;132(2):211-9.

14. Meehan TM, Martinez-Salazar EL, Torriani M. Aftermath of ankle inversion injuries: spectrum of MR imaging findings. Magn Reson Imaging Clin N Am. 2017;25(1):45-61.

15. Hashimoto T, Inokuchi S, Kokubo T. Clinical study of chronic lateral ankle instability: injured ligaments compared with stress X-ray examination. J Orthop Sci. 2009;14(6):699-703.

16. Schneiders A, Karas S. The accuracy of clinical tests in diagnosing ankle ligament injury. Eur J Phys. 2016;18(4):245-53.

17. Guillo S, Bauer T, Lee JW, Takao M, Kong SW, Stone JW, Mangone PG, Molloy A, Perera A, Pearce CJ, et al. Consensus in chronic ankle instability: aetiology, assessment, surgical indications and place for arthroscopy. Orthop Traumat Surg Res. 2013;99(8 Suppl):S411-9.

18. Michels F, Pereira H, Calder J, Matricali G, Glazebrook M, Guillo S, Karlsson J, Acevedo J, Batista J, Bauer T, et al. Searching for consensus in the approach to patients with chronic lateral ankle instability: ask the expert. Knee Surg Sports Traumatol Arthros. 2017. https://doi.org/10.1007/s00167-017-4556-0.

19. Kwon DG, Sung KH, Chung CY, Park MS, Kim TW, Lee SH, Lee KM Associations between MRI findings and symptoms in patients with chronic ankle sprain. J Foot Ankle Surg. 2014;53(4):411-4.

20. Radwan A, Bakowski J, Dew S, Greenwald B, Hyde E, Webber N. Effectiveness of ultrasonography in diagnosing chronic lateral ankle instability: a systematic review. Int J Sports Phys Ther. 2016;11(2):164-74.

21. Jolman S, Robbins J, Lewis L, Wilkes M, Ryan P. Comparison of magnetic resonance imaging and stress radiographs in the evaluation of chronic lateral ankle instability. Foot Ankle Int. 2017;38(4):397-404.

22. Takao M, Uchio Y, Naito K, Fukazawa I, Ochi M. Arthroscopic assessment for intra-articular disorders in residual ankle disability after sprain. Am J Sports Med. 2005;33(5):686-92.

23. Jung $H G$, Kim NR, Kim TH, Eom JS, Lee DO. Magnetic resonance imaging and stress radiography in chronic lateral ankle instability. Foot Ankle Int. 2017;38(6):621-6. 
24. Whiting PF, Rutjes AW, Westwood ME, Mallett S, Deeks JJ, Reitsma JB, Leeflang MM, Sterne JA, Bossuyt PM. QUADAS-2: a revised tool for the quality assessment of diagnostic accuracy studies. Ann Intern Med. 2011:155(8):529-36.

25. Deeks JJ, Altman DG. Diagnostic tests 4: likelihood ratios. BMJ (Clinical research ed). 2004:329(7458):168-9.

26. Deeks JJ. Systematic reviews in health care: systematic reviews of evaluations of diagnostic and screening tests. BMJ (Clinical research ed). 2001;323(7305):157-62

27. Park HJ, Lee SY, Park NH, Kim E, Chung EC, Kook SH, Lee JW. Usefulness of the oblique coronal plane in ankle MRI of the calcaneofibular ligament. Clin Radiol. 2015;70(4):416-23.

28. Park HJ, Lee SY, Park NH, Rho MH, Chung EC, Park JH, Park SJ. Threedimensional isotropic T2-weighted fast spin-echo (VISTA) ankle MRI versus two-dimensional fast spin-echo T2-weighted sequences for the evaluation of anterior talofibular ligament injury. Clin Radiol. 2016;71(4):349-55.

29. Park HJ, Lee SY, Choi YJ, Hong HP, Park SJ, Park JH, Kim E. 3D isotropic T2-weighted fast spin echo (VISTA) versus 2D T2-weighted fast spin echo in evaluation of the calcaneofibular ligament in the oblique coronal plane. Clin Radiol. 2017;72(2):176 e171-7.

30. Scranton PE, McDermott JE, Rogers JV. The relationship between chronic ankle instability and variations in mortise anatomy and impingement spurs. Foot Ankle Int. 2000;21(8):657-64.

31. O'Neill PJ, Van Aman SE, Guyton GP. Is MRI adequate to detect lesions in patients with ankle instability? Clin Orthop Relat Res. 2010;468(4):1115-9.

32. Park HJ, Cha SD, Kim HS, Chung ST, Park NH, Yoo JH, Park JH, Kim JH, Lee TW, Lee $\mathrm{CH}$, et al. Reliability of MRI findings of peroneal tendinopathy in patients with lateral chronic ankle instability. Clin Orthop Surg. 2010;2(4):237-43.

33. Chun KY, Choi YS, Lee SH, Kim JS, Young KW, Jeong MS, Kim DJ. Deltoid ligament and tibiofibular syndesmosis injury in chronic lateral ankle instability: magnetic resonance imaging evaluation at 3T and comparison with arthroscopy. Korean J Radiol. 2015;16(5):1096-103.

34. Yi J, Cha JG, Lee YK, Lee BR, Jeon CH. MRI of the anterior talofibular ligament, talar cartilage and os subfibulare: comparison of isotropic resolution $3 \mathrm{D}$ and conventional $2 \mathrm{D}$ T2-weighted fast spin-echo sequences at 3.0 T. Skelet Radiol. 2016:45(7):899-908.

35. Cha SD, Kim HS, Chung ST, Yoo JH, Park JH, Kim JH, Hyung JW. Intraarticular lesions in chronic lateral ankle instability: comparison of arthroscopy with magnetic resonance imaging findings. Clin Orthop Surg. 2012;4(4):293-9.

36. Chandnani VP, Harper MT, Ficke JR, Gagliardi JA, Rolling L, Christensen KP, Hansen MF. Chronic ankle instability: evaluation with MR arthrography, MR imaging, and stress radiography. Radiology. 1994;192(1):189-94.

37. Cheng Y, Cai $Y$, Wang Y. Value of ultrasonography for detecting chronic injury of the lateral ligaments of the ankle joint compared with ultrasonography findings. Br J Radiol. 2014;87(1033):20130406.

38. Cho JH, Lee DH, Song HK, Bang JY, Lee KT, Park YU. Value of stress ultrasound for the diagnosis of chronic ankle instability compared to manual anterior drawer test, stress radiography, magnetic resonance imaging, and arthroscopy. Knee Surg Sports Traumatol Arthrosc. 2016;24(4):1022-8.

39. Chou MC, Yeh LR, Chen CK, Pan HB, Chou YJ, Liang HL. Comparison of plain $M R I$ and MR arthrography in the evaluation of lateral ligamentous injury of the ankle joint. J Chin Med Assoc. 2006;69(1):26-31.

40. Hua Y, Yang Y, Chen S, Cai Y. Ultrasound examination for the diagnosis of chronic anterior talofibular ligament injury. Acta Radiol. 2012:53(10):1142-5.

41. Joshy S, Abdulkadir U, Chaganti S, Sullivan B, Hariharan K. Accuracy of MRI scan in the diagnosis of ligamentous and chondral pathology in the ankle. Foot Ankle Surg. 2010;16(2):78-80

42. Kim YS, Kim YB, Kim TG, Lee SW, Park SH, Lee HJ, Choi YJ, Koh YG. Reliability and validity of magnetic resonance imaging for the evaluation of the anterior talofibular ligament in patients undergoing ankle arthroscopy. Arthroscopy. 2015;31(8):1540-7.

43. Kumar V, Triantafyllopoulos I, Panagopoulos A, Fitzgerald S, Niekerk LV. Deficiencies of MRI in the diagnosis of chronic symptomatic lateral ankle ligament injuries. Foot Ankle Surg. 2007;13(4):171-6.

44. Lee MH, Cha JG, Lee YK, Choi GC, Paik SH, Lee HK, Park SJ, Kim HJ. The bright rim sign on MRI for anterior talofibular ligament injury with arthroscopic correlation. AJR Am J Roentgenol. 2012;198(4):885-90.

45. Oae K, Takao M, Uchio Y, Ochi M. Evaluation of anterior talofibular ligament injury with stress radiography, ultrasonography and MR imaging. Skelet Radiol. 2010;39(1):41-7.
46. Park HJ, Cha SD, Kim SS, Rho MH, Kwag HJ, Park NH, Lee SY. Accuracy of MRI findings in chronic lateral ankle ligament injury: comparison with surgical findings. Clin Radiol. 2012;67(4):313-8.

47. Staats K, Sabeti-Aschraf M, Apprich S, Platzgummer H, Puchner SE, Holinka J, Windhager R, Schuh R. Preoperative MRI is helpful but not sufficient to detect associated lesions in patients with chronic ankle instability. Knee Surg Sports Traumatol Arthrosc. 2017. https://doi.org/10.1007/s00167-0174567-x

48. Sugimoto K, Takakura Y, Samoto N, Nakayama S, Tanaka Y. Subtalar arthrography in recurrent instability of the ankle. Clin Orthop Relat Res. 2002:394:169-76.

49. Tan DW, Teh DJW, Chee YH. Accuracy of magnetic resonance imaging in diagnosing lateral ankle ligament injuries: a comparative study with surgical findings and timings of scans. Asia Pac J Sport Med Arthrosc Rehabil Technol. 2017:7:15-20.

50. Richardson ML. The Zombie plot: a simple graphic method for visualizing the efficacy of a diagnostic test. AJR Am J Roentgenol. 2016; 207(4):W43W52.

51. Liu K, Gustavsen G, Royer T, Wikstrom EA, Glutting J, Kaminski TW. Increased ligament thickness in previously sprained ankles as measured by musculoskeletal ultrasound. J Athl Train. 2015;50(2):193-8.

52. Croy T, Saliba SA, Saliba E, Anderson MW, Hertel J. Differences in lateral ankle laxity measured via stress ultrasonography in individuals with chronic ankle instability, ankle sprain copers, and healthy individuals. J Orthop Sports Phys Ther. 2012;42(7):593-600.

53. Lee KT, Park YU, Jegal H, Park JW, Choi JP, Kim JS. New method of diagnosis for chronic ankle instability: comparison of manual anterior drawer test, stress radiography and stress ultrasound. Knee Surg Sports Traumatol Arthrosc. 2014;22(7):1701-7.

54. Martella I, Azzali E, Milanese G, Pratico FE, Ruggirello M, Trunfio V, Parziale R, Corrado M, Della Casa G, Capasso R, et al. MRI in acute ligamentous injuries of the ankle. Acta bio-medica : Atenei Parmensis. 2016;87(Suppl 3):13-9.

55. van Putte-Katier N, van Ochten JM, van Middelkoop M, Bierma-Zeinstra SM, Oei EH. Magnetic resonance imaging abnormalities after lateral ankle trauma in injured and contralateral ankles. Eur J Radiol. 2015;84(12):2586-92.

56. Choi WJ, Lee JW, Han SH, Kim BS, Lee SK. Chronic lateral ankle instability: the effect of intra-articular lesions on clinical outcome. Am J Sports Med. 2008:36(11):2167-72.

57. Tao H, Hu Y, Qiao Y, Ma K, Yan X, Hua Y, Chen S. T2-mapping evaluation of early cartilage alteration of talus for chronic lateral ankle instability with isolated anterior talofibular ligament tear or combined with calcaneofibular ligament tear. J Magn Reson Imaging. 2017; 47(1):69-77.

58. Tourne $Y$, Besse JL, Mabit C. Chronic ankle instability. Which tests to assess the lesions? Which therapeutic options? Orthop Traumatol, Surg Res. 2010;96(4):433-46.

59. Ferran NA, Oliva F, Maffulli N. Ankle instability. Sports Med Arthrosc Rev. 2009;17(2):139-45

60. Frost SC, Amendola A. Is stress radiography necessary in the diagnosis of acute or chronic ankle instability? Clin J Sport Med. 1999;9(1):40-5.

61. McCaskie AW, Gale DW, Finlay D, Allen MJ. Chronic ankle instability: the value of talar tilt under general anaesthesia. Br J Sports Med. 1995;29(2):103-4.

62. Jeong BO, Kim TY, Song WJ. Effect of preoperative stress radiographic findings on radiographic and clinical outcomes of the modified Brostrom procedure for chronic ankle instability. J Foot Ankle Surg. 2016:55(1):125-8.

63. Trnka HJ, Ivanic G, Trattnig S. Arthrography of the foot and ankle. Ankle and subtalar joint. Foot Ankle Clin. 2000;5(1):49-62. vi.

64. Samoto N, Sugimoto K, Takaoka T, Fujita T, Kitada C, Takakura Y. Comparative results of conservative treatments for isolated anterior talofibular ligament (ATFL) injury and injury to both the ATFL and calcaneofibular ligament of the ankle as assessed by subtalar arthrography. J Orthop Sci. 2007;12(1):49-54.

65. Kopkow C, Freiberg A, Kirschner S, Seidler A, Schmitt J. Physical examination tests for the diagnosis of posterior cruciate ligament rupture: a systematic review. J Orthop Sports Phys Ther. 2013;43(11):804-13. 